\title{
Ecos de la disputa del antisemitismo en la filosofía jurídico-política de Hermann Cohen
}

\author{
Héctor Óscar Arrese Ígor* \\ Universidad Nacional de La Plata, Argentina
}

\begin{abstract}
En este trabajo intento mostrar que la idea del Estado que Cohen defiende se funda en la integración de las tradiciones judía y alemana. En primer lugar, analizo la tesis de Cohen respecto de la influencia mutua de las dos culturas a lo largo de la historia alemana, expuesta en su conferencia Deutschtum und Judentum (1915). Finalmente intento mostrar la presencia de esta tesis en las ideas de la autoconciencia y del Estado de la Ethik des reinen Willens.
\end{abstract}

Palabras clave: ética, neokantismo, Alemania, judaísmo, autoconciencia, estado

\section{ECHOES OF THE DISPUTE ABOUT THE ANTISEMITISM IN HERMANN COHEN'S JURIDICAL AND POLITICAL PHILOSOPHY}

In this paper I try to show that the idea of the state, that Cohen defends, is based in the integration of the jewish and german traditions. In the first place, I analyze the thesis of the mutual influence of the two cultures along the german history, exposed in his conference Deutschtum und Judentum (1915). Finally, I try to show the presence ot these thesis in the ideas of the selfconciousness and of the state of the Ethik des reinen Willens.

Key words: ethics, neo-kantianism, Germany, judaism, selfconciousness, state

* Doctor en Filosofía de la Universidad Nacional de La Plata (República Argentina). Docente de la misma universidad. 
$\mathrm{H}$

ermann Cohen ha elaborado una teoría del Estado que fue de gran inspiración y guía para el ala revisionista de la socialdemocracia alemana de fines del siglo XIX. Sin embargo, las diferentes interpretaciones que ha recibido su teoría no dejan totalmente en claro los motivos y razones que llevaron a Cohen a sostener su ideal de un Estado socialista, es decir una forma de democracia deliberativa fundada en un cierto igualitarismo en lo económico y en el acceso universal a la educación. Los principales estudiosos de la obra de Cohen acentúan el rol que jugó su experiencia como miembro de la minoría judía, excluida en gran medida de la vida pública en tiempos de Bismarck. Desde este punto de vista, Cohen habría defendido la idea de un Estado inclusivo y tolerante de las diferencias, como una estrategia para defender a sus hermanos de fe y procedencia. ${ }^{1}$

Es conocida la impugnación de Cohen del sistema prusiano de votación por clases (Dreiklassenwahlrecht), que es consecuencia lógica de su defensa encendida del sufragio universal, considerado como conditio sine qua non para la realización de toda democracia deliberativa, porque nadie puede saberse representado por legisladores a quienes no ha podido elegir. En este marco, P. Schmid sostiene la misma hipótesis que H. Holzhey (1993), E. Winter (1980), H. Lübbe (1958) y (1963), así como S. Schwarzschild (1956). Con sus propias palabras:

Como judío emancipado, Cohen reconoce al Estado como la única posibilidad para los judíos de convertirse en miembros plenamente valiosos de la comunidad constituida legalmente. El elogio que hace Cohen del Estado es el elogio de un Estado en el cual las minorías étnicas y religiosas pueden convertirse en ciudadanos que tienen el mismo valor que los demás. Este Estado es el lugar de la emancipación precisamente de los judios, porque 'sin ley no hay ninguna personalidad libre' (E, 118). De alli que el Cohen judío ponga su esperanza en el Estado de derecho prusiano y en su evolución, porque este mismo otorgó a los judíos la plena ciudadanía desde la misma fundación del reino. ${ }^{2}$

Estas lecturas no llevan poca parte de razón, si consideramos el clima político y cultural en el que Cohen tuvo que vivir y desarrollar su obra. Cohen experimentó en carne propia los efectos de la furiosa ola de antisemitismo que se impuso en la Alemania de fines de 1870.

\section{La disputa del antisemitismo}

Para hacerse una idea de la atmósfera opresiva en la que vivían los judíos, basta con considerar algunos sucesos desagradables que ocurrieron en aquel entonces. Por

1 Ver. Holzhey, (1993), 27-28; Winter, (1980), 333-334. Acerca de la superación de las pretensiones hegemónicas de la religión en la universalidad del Estado, Ver. Lübbe, (1958), 234; (1963), 240. Para la cuestión de la neutralidad estatal, ver Schwarzschild, (1956), 216.

2 "Als emanzipierter Jude erkennt Cohen im Rechtsstaat die einzige Möglichkeit der Juden, vollwertige Mitglieder der rechtlich verfassten Gemeinschaft zu werden. Cohens Staatslob ist das Lob für einen Staat, in dem die religiösen und ethnischen Minderheiten vollwertige Bürger werden können. Dieser Staat ist der Ort der Emanzipation gerade der Juden, denn ,ohne Gesetz keine Freiheit, und ohne die im Gesetz bestehende Gemeinschaft keine freie Persönlichkeit' (E, 118). Daher setzt der Jude Cohen seine Hoffnung auf den preussischen Rechtsstaat und auf dessen Entwicklung, weil dieser seit der Reichsgründung den Juden die volle Staatsbürgerschaft zugestand“ (Schmid, 1993, 77). 
ejemplo, Treitschke declaró que le parecía indignante que continuamente llegaran judíos de Polonia que sólo se dedicaban a vender ropa, pero que con el tiempo llegarían a dominar los periódicos y las bolsas de valores del país. Algunas organizaciones políticas pedían públicamente que se les quitara a los judíos el derecho al sufragio, se les examinara periódicamente para renovar su permiso de trabajo, se los obligara a bautizarse según el ritual cristiano, etc. ${ }^{3}$

Este odio fue tomando forma a partir de la acción de organizaciones políticas tales como el Christlichsoziale Partei, fundado por el predicador Adolf Stoecker. También en Marburg florecieron organizaciones estudiantiles antisemitas, tales como la Verein deutscher Studenten (fundada a fines de 1880), o la organización de Otto Böckels, sumamente poderosa en aquel entonces. Ejerció mucha influencia sobre los estudiantes la publicación del panfleto de Böckels Die Juden, die Könige unserer Zeit (Los judíos, los reyes de nuestro tiempo), que fue distribuido por los mismos estudiantes. ${ }^{4}$

Cohen mismo se vio obligado a participar, en calidad de perito, de un proceso judicial contra un acto de antisemitismo. Se trató de la demanda del rabino Leo Munk contra el docente Ferdinand Fenner, quien en una alocución de mitin afirmó que el Talmud exige a los judíos que traicionen a los cristianos. Del lado de los antisemitas fue convocado como perito el orientalista Paul de Lagarde (de Göttingen), mientras que por parte de la comunidad judía fue llamado Cohen. Contra la débil y escasamente informada argumentación de Lagarde, Cohen sostuvo, apoyándose en citas de los textos sagrados, que la religión judía es una religión de la humanidad y no ya de una nación particular. Por lo tanto, el Talmud jamás podría prescribir que se traicionara a los extraños, sean estos cristianos o de otra religión. El proceso culminó con la condena de Fenner a dos semanas de prisión más el pago de las costas del juicio. ${ }^{5}$

Como bien apunta Palmer, el caso de Fenner estuvo mal planteado judicialmente desde el comienzo. La cuestión no era si la religión judía permitía o no que se estafara o robara a los cristianos, sino más bien el problema consistía en si Fenner había insultado o no a la comunidad judía en su discurso. De allí la improcedencia de la consulta a Cohen y a de Lagarde, en calidad de peritos. ${ }^{6}$

Pero la cuestión judía alcanzó el status de debate académico recién con ocasión del artículo de Treitschke, "Unsere Aussichten" ("Nuestras perspectivas"), publicado en 1879 en los Preussische Jahrbücher (Anales prusianos). Allí Treitschke, preocupado por la masiva inmigración de judíos polacos, exigía la asimilación total de los judíos a la Alemania cristiana de la época. En este artículo llegó a afirmar que ellos son los causantes de todas las desgracias de la Alemania de la época ("die Juden sind unser Unglück" $)^{7}$. Pocos intelectuales tomaron posición contra Treitschke, lo cual puso en

3 Geismann, (1993), nota al pie de la 370.

4 Sieg, (1994), 154.

5 Sieg, (1994), 156-157.

6 Palmer, (2006), 50-53. 
evidencia el rechazo que existía frente a las tradiciones y la cultura judía.

Cohen reaccionó con vehemencia frente al artículo de Treitschke con su "Bekenntnis in der Juden Frage" ("Declaración sobre la cuestión judía", 1880), donde negaba toda diferencia substancial entre el judaísmo y el cristianismo. La semejanza entre ambas religiones se fundaba, en opinión de Cohen, en su profesión de fe en un único Dios, garantía de la moralidad. El rasgo definitorio del judaísmo sería la legalidad inherente a su religiosidad, cuyo ejemplo paradigmático es la Torá misma. Justamente la valoración de la legalidad sería también, en opinión de Cohen, un elemento central de la tradición y la cultura alemanas. ${ }^{8}$ Cohen defendía la germanidad de los judíos y minimizó el peligro del antisemitismo, lo cual le valió el reproche de sus hermanos de fe. Cohen propuso incluso que el descanso sabático sea trasladado al día domingo, para integrar de algún modo el calendario judío al de la comunidad cristiana; esta propuesta provocó un enérgico rechazo en su comunidad. ${ }^{9}$

Contra la intención de Cohen de fusionar el judaísmo y la germanidad, Rosenzweig malinterpretó su Religion der Venunft aus den Quellen des Judentums (Religión de la razón a partir de las fuentes del judaísmo), afirmando que allí Cohen rompió con el velo del idealismo y abandonó la búsqueda de la sistematicidad, reemplazándola por los sentimientos nacionales y religiosos judíos. ${ }^{10}$ Por el contrario, Cohen intentó en algunos escritos de circunstancias refutar la teoría de que los judíos alemanes pertenecerían a una raza diferente de las de sus conciudadanos, a partir de consideraciones tales como el efecto homogeneizante que tuvo sobre el organismo de todos los alemanes, incluidos los judíos, el hecho de haber vivido en las mismas condiciones climáticas durante tantos siglos. ${ }^{11}$

Pero es necesario admitir que Cohen nunca defendió la política de asimilación violenta que pedían los antisemitas. En un texto publicado en 1890, es decir 10 años después del Antisemitismusstreit con Treitschke, Cohen se oponía enérgicamente a la conversión compulsiva de los judíos al cristianismo. Esto significaría, argumentaba Cohen, una traición a la fidelidad a la propia fe y a las propias raíces culturales. ${ }^{12} \mathrm{Su}$ lucha por la preservación de la identidad judía no fue obstáculo para que se opusiera al sionismo de Buber. Contra éste, consideraba que la esencia del judaísmo es su carácter itinerante, que es la causa de su universalidad moral característica, e implica que la Palestina de todo judío es el mundo entero y no un territorio particular. ${ }^{13}$

Cassirer tuvo que enfrentar también una Antisemitismusstreit. El redactor responsable de los Kant-Studien, Bruno Bauch, publicó en 1917 en esta revista un artículo sobre el

7 Sieg, (1994), 148.

8 Wiedebach, (1997), 47-48.

9 Sieg, (1994), 151-152.

10 Zank, (2006), 1-2.

11 Wiedebach, (1997), 42.

12 Wiedebach, (1997), 44.

13 Wiedebach, (1997), 35. 
concepto de nación (“Über den Begriff der Nation”), donde advertía respecto del peligro que representaba la comunidad judía para el mantenimiento de la unidad de la cultura alemana. El carácter nacional, sostenía Bauch, se fija ante todo en el suelo y la sangre (Boden und Blut). Cassirer escribió un artículo polémico que no fue publicado en la revista, y por esta razón solicitó la retractación pública de Bauch.

Dado que la retractación nunca tuvo lugar, Cohen y Cassirer abandonaron la Sociedad Kant, a lo que le siguió la retirada de otros miembros. Finalmente, Bauch debió renunciar a su cargo en la revista, no sin antes victimizarse en una nota publicada en enero de 1917 en el periódico Panther, donde se presentó como el blanco de la censura judía. Bauch fue apoyado por varios colegas, y el mismo Heimsoeth le escribió a Hartmann en febrero de 1917 que opinaba que los Kant-Studien se habían finalmente judaizado. ${ }^{14}$

Además Cassirer tuvo serios problemas para ingresar a la carrera académica a causa de su condición de judío. Windelband decidió no apoyar su nombramiento en Strassburg precisamente por esta causa. De allí que Natorp le escribiera a Görland en 1902, que le parecía deprimente tener que dar la razón a Cohen acerca del antisemitismo imperante, cuando se esforzaba permanentemente por calmarlo, intentando convencerlo de lo contrario. ${ }^{15}$

Pero la oposición de Cohen al nacionalismo extremo convivió en él con un ardiente patriotismo. En medio de la Primera Guerra Mundial tuvo lugar en Alemania un movimiento de ideas que legitimó la guerra en base a la superioridad cultural, política y moral alemana sobre los países aliados. Por eso se la denominó como la "guerra de los espíritus" (Krieg der Geister). Cohen participó activamente de este movimiento nacionalista, orientado a levantar el ánimo de la población sumida en la guerra, sobre todo con el dictado de una serie de conferencias en diversos centros intelectuales, inclusive en Marburg, de donde se había retirado ya en 1912.

Entre ellas, fue famosa su "Über das Eigentümliche des deutschen Geistes" ("Sobre la particularidad del espíritu alemán"), pronunciada en la Sociedad Kant de Berlín en octubre de 1914. Las "ideas de 1914", como también se denominó a este movimiento, en realidad encerraban una notable heterogeneidad de puntos de vista. Estos iban desde la estimación de la "profundidad" de la cultura alemana (cimentada sobre el cultivo de la interioridad, desde Lutero en adelante), frente a la superficialidad de la cultura anglosajona (utilitarista y empirista), hasta la idea de la superioridad del socialismo alemán por sobre la democracia liberal de mayorías propia de los ideales del oeste.

En este último caso, se valoraba la idea socialdemócrata de la formación moral del ciudadano para una identificación con los objetivos del Estado y la obediencia

14 Sieg, (1994), 400-402.

15 Sieg, (1994), 335. 
consciente y responsable de sus leyes, frente a la idea liberal de un egoísmo universal, con su pragmatismo consecuente en el ejercicio de la ciudadanía. ${ }^{16}$ Cohen, al igual que otros judíos, se entusiasmaba con la idea de que la participación activa de los judíos en la guerra constituía una muestra clara de patriotismo, que haría posible la supresión de las barreras que el antisemitismo había puesto a su integración a la vida de la nación. De allí su oposición decidida al sionismo, como una corriente que buscaba aislar a la comunidad judía del resto de los alemanes. En 1914 firmó junto con 300 notables judíos una declaración del "Comité Anti-sionista". Cohen estaba embargado de tal optimismo, que llegó a solicitar a la oficina de relaciones exteriores en 1915 apoyo financiero para dictar una serie de conferencias en EEUU, con el fin de mejorar la imagen de Alemania en el extranjero, mostrando la civilidad, la libertad y la humanidad que reinaban en la nación. ${ }^{17}$

Cohen justificó también la participación de Alemania en la Primera Guerra Mundial, presentándola como una guerra para acabar con las causas de la guerra en general. Es decir, como una forma de establecer el reino de la razón sobre el mundo, misión que le estaba históricamente reservada a Alemania por su tradición humanista, de Lutero y Kant en adelante. Cohen veía a Alemania como la nación que puede realizar el ideal moral universalista kantiano, y consideraba que esto la convertiría en la educadora de la humanidad. ${ }^{18}$

Esta actitud positiva que Cohen demostró permanentemente frente a la nación alemana estaba acompañada del deseo de que su comunidad judía sea finalmente integrada como miembro de pleno derecho. De allí que su defensa de la idea de nación estuviera matizada con el rechazo de un nacionalismo excluyente que divorciara germanidad y judaísmo para siempre.

De lo que se ha argumentado hasta aquí puede verse que las interpretaciones que he mencionado más arriba han señalado con acierto la necesidad que experimentó Cohen de dar forma teórica a su lucha por la equiparación de derechos del pueblo judío y el resto de los alemanes. Sin embargo, considero que esta lectura no toma en consideración aspectos de tipo sistemático-filosófico que están a la base de la teoría coheniana del Estado. Es de destacar que el estudio de H. Wiedebach (1997) ha profundizado en el rol que juega el concepto de nación en esta teoría, sobre todo en relación a la virtud de la humanidad, que constituye un puente entre la ética y la estética.

En este trabajo me propongo ir más allá del valioso trabajo de $\mathrm{H}$. Wiedebach, sondeando la concepción que Cohen tenía de las relaciones entre la germanidad y el judaísmo, para luego reconstruir las consecuencias filosóficas que de allí se desprenden. En particular, me centraré en el análisis del idealismo alemán, entendido

16 Sieg, (1994), 375-377.

17 Sieg, (1994), 394-397.

18 Wiedebach, (1997), 22, 129-131. Curiosa interpretación, por cierto, de los escritos de Kant sobre la Paz Perpetua y del abierto cosmopolitismo de su propuesta. 
como un aporte del judaísmo a la germanidad. Esto significa que la ética kantiana, y el ideal socialista que Cohen toma de ella, serían la culminación de la integración entre la germanidad y el judaísmo, y su fruto filosófico más maduro. Con esta tarea en mente, intentaré mostrar que la teoría del Estado de la Ethik des reinen Willens no responde a intereses puramente estratégico-instrumentales de Cohen, en orden a proteger a su comunidad judía de toda suerte de atropellos y exclusiones, sino que es parte de una concepción más profunda y compleja sobre el rol histórico y cultural que han jugado la cultura judía y la germanidad.

\section{La germanidad y el judaísmo}

Cohen pronunció en 1915 la conferencia "Germanidad y Judaísmo" ("Deutschtum und Judentum"), con ocasión del rebrote de antisemitismo en la Alemania de la Gran Guerra, en la que, paradójicamente, los judíos con los demás alemanes peleaban codo a codo en las trincheras. ${ }^{19}$ Por lo tanto, el texto está dirigido tanto contra los antisemitas como contra los sionistas, que negaban también toda comunidad a los judíos y los alemanes, en nombre de la nación originaria a la que el pueblo israelita debía retornar. ${ }^{20}$

En este texto, Cohen constata que, ya desde sus primeros momentos, la germanidad estuvo íntimamente unida con el judaísmo. En tiempos de la antigua Roma, dice Cohen, los judíos ya se habían asentado en las riberas del Rin y tomaron parte en la vida de la primitiva Germania. En los siglos posteriores se fundaron en territorio alemán las principales escuelas judías (las de Mainz, Worms, Speyer, entre otras), que ejercieron una influencia incomparable sobre los centros judíos de todo el mundo, en especial de Europa y América. ${ }^{21}$

La vinculación del judaísmo con la germanidad fue tan intensa que resistió a las persecuciones crueles y a las expulsiones sucesivas del territorio alemán en tiempos de la peste negra. En efecto, una vez transcurridos algunos años luego de su expulsión, los judíos retornaban a su patria alemana. La participación de los judíos en la poesía medieval, tanto en la poesía mística como en el Minnesang, pone en evidencia esta conexión con el espíritu del pueblo en que se habían asentado. ${ }^{22}$ Esta unión se profundizó con la obra de Moses Mendelssohn, quien eligió a la lengua alemana para dar continuidad al judaísmo y preservar su doctrina. En una época se llegó a afirmar que la doctrina judía viene de Alemania, tanto como antes había provenido de Sión. ${ }^{23}$

Pero la unión profunda entre el judaísmo y la germanidad se ha efectivizado sobre todo gracias a la Reforma luterana y al idealismo alemán. Cohen determina a la germanidad y al judaísmo a partir del análisis de una cultura común a estas dos,

19 DuJ, 292-293.

20 DuJ, 291; 299-301.

21 DuJ, 253-254.

${ }^{2} \mathrm{DuJ}, 254$.

${ }^{23} \mathrm{DuJ}, 260$. 
que de algún modo las une y les confiere una íntima afinidad. Se trata de la cultura griega, y en especial de la filosofía platónica. El punto de contacto entre el judaísmo y la filosofía griega puede ubicarse en épocas del exilio voluntario del judaísmo en Alejandría. Filón de Alejandría perteneció a aquel movimiento de judíos alejandrinos que buscaban una síntesis entre ambas culturas. Filón tomó del platonismo el concepto de Logos, entendido como la mediación entre Dios y el hombre. ${ }^{24}$ Ya en este momento fundacional el judaísmo entró en una relación de comunión profunda con el cristianismo, porque este último parte de la afirmación de que Cristo es el Verbo encarnado, es decir el Logos o Palabra de Dios, en la medida en que constituye una mediación entre lo divino y lo humano. ${ }^{25}$

Cohen entiende al concepto de Logos como la razón humana. Tanto el judaísmo como el cristianismo sostienen, desde el punto de vista de Cohen, que sólo el ejercicio libre de la razón puede ayudarnos a llegar a Dios. La última raíz del concepto de razón se encuentra en la teoría platónica de las Ideas. Cohen interpreta el concepto platónico de Idea en términos de hipótesis, interpretación que luego hará famosa su discípulo Natorp. ${ }^{26}$ De acuerdo con esta perspectiva, la Idea platónica no puede ser entendida como opuesta al conocimiento sensible, ni como un ente eterno e inmutable, una substancia siempre idéntica a sí misma. ${ }^{27}$ Por el contrario, se trata de la actividad misma de la razón que produce los conceptos y las hipótesis científicas a partir de sí misma. ${ }^{28}$

Este concepto de la razón que pone el origen del conocimiento en sí misma es retomado por Martin Lutero, en su teoría de la justificación de las creencias religiosas. En el contexto de la Reforma Protestante, el creyente debe aceptar sólo aquellas verdades a las que llega por el trabajo autónomo de su propia conciencia. ${ }^{29}$ De allí la exigencia de la libre interpretación de la Biblia, y la discusión de toda la dogmática católica, como una estructura impuesta desde fuera a la conciencia por el poder eclesiástico. ${ }^{30}$

Martin Lutero consideraba que las instituciones eclesiásticas, como por ejemplo el celibato sacerdotal o los diferentes sacramentos, no podían ser aceptados sin más, sino que necesariamente debían pasar por el tamiz crítico de la razón. Es decir, que sólo la razón misma puede presidir el tribunal que juzgue la legitimidad de los poderes establecidos. ${ }^{31}$ Es sabido que sólo algunas instituciones eclesiásticas

${ }^{24} \mathrm{DuI}$, 237. Sobre Filón de Alejandría, ver $\operatorname{ErW}, 111,210$ ss., 335.

${ }^{25} \mathrm{DuJ}, 238$.

${ }^{26}$ Para un estudio exhaustivo de la interpretación particular del concepto platónico de Idea en el neokantismo de Marburg, ver Lembeck, (1994). Para la interpretación del del concepto platónico de Idea ver LrE, 5-7, 13, 15 ss., 20 ss., 65, 87 ss.,94-96, 102, 111, 211 ss., 216, 247, 303, 311-313, 316, 326, 344, 374, 376, 379, 394, 400, 407, 445, 457-460, 482-485, 500, 502 ss., 528, 595.

27 DuI, 239.

${ }^{28}$ DuJ, 239-240. Para el concepto de hipótesis ver LrE, 7 ss, 116, 247, 251 ss., 594, 430-433, 440 ss., 448 ss., 504, 547 ss., 601.

${ }^{29} \mathrm{DuJ}, 241$. Ver ErW, 301 ss., 307, 403, 516.

${ }^{30} \mathrm{DuI}, 242$.

${ }^{31}$ Esta afirmación tiene sus raíces en la concepción del logos y la idea como justificación. Ver LrE, 20, $30,317,528$. 
sobrevivieron al examen de la conciencia luterana. Cohen considera que, de este modo, el Protestantismo trae por primera vez al espíritu auténtico de la germanidad a su realidad histórica, porque la germanidad puede ser entendida sólo como una cultura idealista. ${ }^{32}$ Esto quedará en evidencia si se recorre la historia de la filosofía alemana, desde Nicolás de Cusa hasta Kant, pasando por Leibnitz.

Pero en realidad esta idea no es exclusiva del luteranismo, sino que puede remontarse a los textos sagrados del judaísmo, en particular hasta los salmos, que fueron traducidos magistralmente por Lutero. En las fuentes del judaísmo Dios aparece como el Autor del Ser, que invita al hombre a que lo sirva libremente, motivado solamente por el amor a su Creador. ${ }^{33}$ Pero este servicio libre sólo puede ser realizado desde el discernimiento crítico, esto es desde la conciencia vigilante que sólo confía en sí misma. Aquí no tiene la última palabra la certeza de tipo científico, sino más bien aquella que depende de la conciencia moral, porque sólo ésta última puede decidir si tal o cual institución o idea está de acuerdo con el servicio al Dios de la justicia. ${ }^{34}$ Por lo tanto en el judaísmo la ética no queda excluida de la religión como una mera disciplina secular, sino que más bien está integrada en el discernimiento mismo que está a la base de la vida religiosa.

Esto es característico también de la Ilustración alemana, que no seculariza totalmente a la ética sino que la pone en íntima relación con los problemas de la religión, siempre según la lectura de Cohen. ${ }^{35} \mathrm{El}$ concepto mismo de autonomía, que es central en el contexto de la Ilustración, tiene sus raíces en la idea de la pureza del alma, que aparece repetidamente en los salmos. Esta idea consiste en que el hombre nunca puede perder la pureza de su alma, sin importar lo contaminada que pueda estar por sus pecados. ${ }^{36}$ La razón de esto es que el hombre sólo puede ser tal en tanto que conserva su conciencia moral, es decir su capacidad de juicio crítico autónomo.

Por eso en la tradición judía no hay contradicción entre los mandamientos, es decir la Ley de Dios, y la libertad de la conciencia moral de cada uno. Cohen considera que en las fuentes del judaísmo esta implícita la distinción kantiana entre el sujeto meramente individual y aquella persona que se somete voluntariamente a aquella legislación universal que se ha dado a sí misma. ${ }^{37}$ De este modo, la ética kantiana constituye una mediación entre la germanidad y el judaísmo, en una instancia de integración de lo mejor de las dos tradiciones.

La ética kantiana dio vida a lo mejor de las tradiciones alemana y judía, en el ideal del socialismo, que exige el tratamiento de la persona como un fin en sí mismo,

32 DuJ, 241. Para el concepto de idealismo en Cohen, Ver LrE, VIII, XII, 6, 251, 289, 297, 303, 305, 320, 457, 462 ss., 594-, 480, 500 ss., 594-595, 598, 600, 606.

${ }^{3} \mathrm{DuJ}, 244$.

${ }^{34} \mathrm{DuJ}, 242-243$.

${ }^{35} \mathrm{DuJ}, 243$.

${ }^{36} \mathrm{DuJ}, 245$.

$37 \mathrm{DuJ}, 246$. 
y nunca como un medio. ${ }^{38} \mathrm{El}$ ideal judeo-germano del socialismo le permite a Cohen impugnar el capitalismo salvaje, que trata al obrero como un mero medio para el enriquecimiento del empresario o de las clases que gobiernan al Estado. De acuerdo con esto, Cohen propone un sistema de cooperativas, donde los obreros controlen el proceso de producción y puedan tener acceso a una vida digna. ${ }^{39}$ De este modo, Kant entiende a la humanidad como un ideal que trasciende al egoísmo de cada sujeto individual hacia el objetivo de la construcción de una comunidad integrada por todas las personas morales. ${ }^{40}$ La subjetividad común a todos los ciudadanos es el Estado, en cuya vida los individuos toman parte por medio de la legislación. ${ }^{41}$

Este tipo de subjetividad pertenece a la tradición idealista alemana pero está también en consonancia con la tradición bíblica. ${ }^{42}$ Ernst Cassirer ha hecho referencia a esta profunda convicción de su maestro en las palabras siguientes, pronunciadas frente a su tumba el 7 de abril de 1918:

Cuando Moisés se coloca frente a Dios en la zarza ardiente, y le pregunta con qué nombre debe denominarlo a Dios, cuando el pueblo le pregunte cómo se llama el Dios que lo ha enviado, Dios le responde con las siguientes palabras: 'Yo seré, el que soy. Diles que 'yo soy' te ha enviado a ellos'. En esta alta abstracción del 'yo', en esta concepción de la persona apartada de toda cosificación, en esta superación de todo lo sensible y de toda figuración por medio del principio espiritual de la personalidad, se enraíza la fuerza auténtica del monoteísmo y su misión universal. ${ }^{43}$

La cultura alemana representa entonces un momento fundamental en el desarrollo del judaísmo universal mismo; de este modo, Alemania es la patria del alma y la religión de todo judío, tanto como lo es Israel. Esto implica que los judíos de todo el mundo están vinculados por obligaciones de piedad hacia el Estado alemán, como su tierra originaria. ${ }^{44}$ Pero también el Estado alemán está obligado a incluir como ciudadanos de pleno derecho a los judíos que viven dentro de sus fronteras, en

${ }^{38} \mathrm{DuJ}, 263$.

${ }^{39} \mathrm{ErW}, 305$. Cohen retomó las reflexiones de su maestro Lange en torno a la importancia del cooperativismo. Lange había fundado en Duisburg una cooperativa de consumo, previendo la fundación más tarde de una cooperativa de producción. En su periódico, Lange abogó intensamente por un sistema estatal de créditos para financiar tanto las cooperativas obreras como las agrarias. Asimismo, exigió una reforma agraria, a partir de la división de los latifundios pertenecientes a los Junkers (Van der Linden, 1988, 295).Acerca de la influencia de Lange en Cohen en relación al cooperativismo, Ver. Schwarzschild, (1956), 219.

40 Acerca del concepto de humanidad en la ética de Cohen, Ver ErW, 82, 251, 294, 485, 493, 557.

${ }^{41} \mathrm{DuJ}, 270 ; \mathrm{ErW}, 245$.

${ }^{42} \mathrm{DuJ}, 266$. Cohen trata sistemáticamente esta cuestión en $E r W, 55,146,214,235,405,496$.

43 "Als Moses am brennenden Dornbusch vor Gott steht, und als er fragt, welchen Namen Gottes er nennen solle, wenn das Volk ihn frage, wie der Gott heiße, der ihn gesandt habe, da antwortet ihm Gott mit den Worten: 'Ich werde sein, der ich bin. Sage ihnen, der 'Ich bin' hat mich zu euch gesandt'. In dieser letzten und höchsten Abstraktion des 'Ich', in dieser vor allem Dinglichen abgewandten Erfassung der Person, in dieser Überwindung alles Sinnlichen und Bildlichen durch das reine geistige Prinzip der Persönlichkeit wurzelt die eigentliche Kraft des Monotheismus und seine weltgeschichtliche Mission." (Cassirer, 1918, 70).

${ }^{44} \mathrm{D} u J, 274$. 
virtud del aporte substancial que el judaísmo ha hecho a la nacionalidad alemana misma. Esto exige, entre otras cosas, la protección de los judíos que estudian en las universidades alemanas, impidiendo que sean excluidos arbitrariamente de las asociaciones estudiantiles, que por los años en que Cohen enseñaba tenían un claro corte antisemita; tampoco debería permitirse de allí en adelante que los profesores judíos sean insultados y difamados gratuitamente por la comunidad académica. ${ }^{45}$ Será inadmisible también la práctica extendida en aquellos días, consistente en presionar a los judíos para que se conviertan al cristianismo, a cambio de privilegios y favores estatales. ${ }^{46}$

Pero la inclusión de los judíos en la vida estatal es sólo una parte de la inmensa tarea que tiene ante sí el Estado alemán, a la hora de realizar históricamente la idea moral de la humanidad. Más fundamental aún es la eliminación de las asimetrías sociales y económicas, la garantía a todos los ciudadanos alemanes del acceso a la educación, etc. ${ }^{47}$ Es decir, que sólo la realización del Estado socialista llevará a su plenitud tanto a la germanidad como al judaísmo, aunque no podrá tratarse del desarrollo de un Estado tomado aisladamente, sino más bien de una federación de Estados. ${ }^{48}$

A esta comunidad jurídica internacional le está reservada la puesta en práctica del ideal moral de la humanidad. Si el socialismo no es cosmopolita, no será un socialismo en lo absoluto. ${ }^{49}$ Pero esto vale también en sentido contrario, porque una federación de Estados sólo será posible si cada uno de estos Estados está construido de acuerdo con el ideal socialista. La razón de esto es que la guerra es generalmente provocada por intereses de grupos económicos que se apoderan del Estado y lo obligan a responder a sus exigencias. ${ }^{50}$

\section{El problema de la autoconciencia}

Como hemos visto hasta aquí, Cohen se considera como un heredero de la tradición del idealismo alemán, en particular de la filosofía kantiana. Si se toman en cuenta el concepto de germanidad y de judaísmo que he expuesto más arriba, podrá darse con uno de los motivos fundamentales de su adhesión a esta tradición filosófica. Pero Cohen sostiene un concepto particular de idealismo, que es necesario aclarar.

Retomando lo dicho en la sección anterior, el idealismo se caracteriza ante todo porque construye sus teorías a partir de una hipótesis, concepto que Cohen atribuye a Platón. Esto significa que la filosofía sólo puede apoyarse en la actividad autoproductiva del pensamiento, lo que le impide partir de los datos de los sentidos

${ }^{4} \mathrm{DuJ}, 278-279$.

${ }^{46} \mathrm{DuJ}, 280$.

$47 \mathrm{DuJ}, 282-283$.

${ }^{4} \mathrm{DuJ}, 286$.

49 DuJ, 287.

${ }^{\circ} \mathrm{DuJ}, 289-290$. 
o de determinadas tradiciones culturales impuestas por la comunidad a la que uno pertenece o de creencias religiosas fundadas en la mera autoridad eclesiástica. ${ }^{51}$ Por el contrario, la filosofía idealista debe partir de la ciencia, como una construcción fundada en la mera razón. ${ }^{52}$

En el caso de la filosofía teórica, Cohen tiene su fundamento firme e inconmovible en la ciencia matemático-natural de su tiempo, fundada en el cálculo infinitesimal. ${ }^{53}$ Pero a la hora de fundamentar su filosofía práctica, Cohen se enfrenta con una situación más compleja, porque no encuentra un candidato inequívoco al título de ciencia fundamentadora de la ética. La psicología presupone el concepto de humanidad, pero no puede dar cuenta de él por sí misma, por lo que necesita de la ética y no a la inversa.$^{54}$ Lo mismo ocurre con la sociología, que parte de una serie de normas que deben regir la vida social pero no puede tematizarlas sin ayuda de la filosofía moral. ${ }^{55}$ Por otro lado, la historia se ocupa sólo de los grandes hombres y no de toda la humanidad, lo que impide la fundamentación de la ética universalista que Cohen está buscando. ${ }^{56}$

Finalmente, Cohen escoge al derecho como la ciencia fundante de la ética, porque tiene la universalidad y necesidad que esta disciplina filosófica requiere ${ }^{57}$ El derecho se aplica a todos los ciudadanos sin distinción, porque en última instancia es el producto de su actividad legisladora en el Parlamento, por medio de sus representantes en el Poder Legislativo. Es decir, que la ley es el producto de la voluntad libre y la razón práctica de quienes luego se someterán a sus dictados. Pero no es sólo la universalidad del derecho lo que motivó la decisión de Cohen de construir su ética sobre esta ciencia. También ha jugado un rol central su carácter necesario. Este se funda en que, una vez que una ley ha sido promulgada, adquiere independencia respecto de las voluntades empíricas y particulares de los legisladores y los ciudadanos que ellos representan, si bien la ley es el resultado de la expresión de estas mismas voluntades individuales. Es decir, que la voluntad jurídica expresada en esa ley podrá ser alterada sólo por medio de otro acto jurídico, también independiente de los caprichos de las voluntades cambiantes de los ciudadanos (por ejemplo, la anulación de esa ley, o su reemplazo por otra, etc.). Por lo tanto, la ley no podrá ser sino como es, lo que significa que es necesaria. La universalidad y la necesidad de la ciencia del derecho la convierten, a los ojos de Cohen, en algo semejante a la ciencia matemático-natural de la época.

Entonces el derecho debe estar en condiciones de responder al problema

51 Se trata del concepto de fundamentación (Grundlegung). Ver ErW, 85, 266, 284, 428-430, 436, 512.

52 LrE, 19 ss., 23, 425 ss.589, 599. Ver la interpretación de Cohen del método trascendental kantiano en KTE, 93-110.

${ }^{53} \operatorname{LrE}, 32-33,123-125,129,132-135,165$.

${ }^{54} \mathrm{ErW}, 29,40$.

${ }^{5} \mathrm{ErW}, 41$.

${ }^{56} \mathrm{ErW}, 30 \mathrm{ss}$.

57 Por esta razón Cohen denomina al derecho como la matemática de las ciencias sociales ( $E r W, 66$ y 436). 
fundamental de la ética, que es la correlación entre el individuo y la universalidad de la ley. ${ }^{58}$ Para lograr este objetivo, el derecho debe proveer a la ética de alguna forma de subjetividad que haga posible esta relación. Se trata del concepto de autoconciencia, que juega un rol central en la ética de Cohen, quien la busca en el concepto mismo de Estado, entendido como la totalidad del sistema del derecho, porque incluye tanto a quienes producen el derecho como a sus destinatarios..$^{59}$

Sin embargo, no es una empresa fácil fundamentar el concepto del Estado, porque es necesario examinar en primer lugar una categoría que no se aviene del todo con el concepto idealista del derecho que Cohen defiende. Se trata de la figura de la comunidad, que revestía una gran importancia en la jurisprudencia de la época en que Cohen redactó su ErW. Para hacerse una idea del influjo ejercido por esta noción baste con recordar que von Savigny fundamentó al Estado en el espíritu del pueblo (Volksgeist), es decir en una comunidad prepolítica de lenguaje, tradiciones culturales, etc. ${ }^{60}$ Por eso Cohen consideró inevitable discutir el concepto de comunidad, que tiene de por sí un carácter extrajurídico, para poder finalmente fundamentar al Estado en el pensamiento puro de la ciencia del derecho. De este modo, Cohen intentó arribar a un concepto idealista de Estado, que esté en comunión con lo mejor de la germanidad y el judaísmo, tradiciones con las que se identificaba profundamente, según vimos en la sección anterior.

\section{La crítica del concepto de comunidad}

Este punto muestra inequívocamente que la motivación principal de Cohen, para adoptar el ideal del Estado democrático de derecho, estaba dada por su necesidad de fundamentarlo en la tradición idealista y kantiana, que él consideraba de ascendencia germano-judaica. Es decir, que el examen de su discusión del concepto de comunidad puede dejar en evidencia que Cohen tenía razones más profundas para defender un Estado de cuño universalista que la mera necesidad de garantizar la inclusión de su pueblo judío minoritario en la Alemania de aquel entonces. En lo que sigue intentaré reconstruir los momentos fundamentales de la argumentación de Cohen en este punto. ${ }^{61}$

En primer lugar, el concepto de comunidad supone aquello que Cohen denomina como el prejuicio de la particularidad, es decir la afirmación de que lo particular puede ser conocido con independencia del concepto universal. ${ }^{62}$ Se trata de la tesis de que el concepto universal es el producto de la suma de los elementos individuales

\footnotetext{
${ }^{5} \mathrm{ErW}, 65$.

${ }^{59}$ Dicho de otro modo, el ideal de Cohen significa, como afirma Schwarzschild, que "eventualmente la distinción entre los que ponen las reglas y aquellos que las obedecen puede ser eliminada, que las personas pueden darse las reglas a sí mismas en una democracia completa" ("eventually the distinction between ruler and ruled will be obliterated, that the people will rule themselves in a complete democracy"; Schwarzschild, 1956, 219)."

${ }^{60}$ Cohen criticó explicitamente la teoría de von Savigny en ErW, 250 ss.

${ }^{61}$ Cohen se ha ocupado del concepto de comunidad en repetidas ocasiones a lo largo de su $\operatorname{ErW}$ (76, 219, 237-239, 241, 249-250, 256, 268 ss., 433, 484, 489, 576).

${ }^{62} \mathrm{ErW}, 234$.
} 
que, en primer lugar, se han observado empíricamente. Es decir que quienes defienden el concepto de comunidad como el medio para la realización de la autoconciencia, en última instancia afirman que lo particular es lo único verdadero, y que lo universal es un mero derivado suyo.

La comunidad es el concepto de una totalidad, que no representa un salto cualitativo respecto de las particularidades que la integran, sino que es simplemente la unidad de la multiplicidad de todas ellas. Por ejemplo, el concepto de tal o cual nación como una comunidad, no es otra cosa, según Cohen, que el de un conjunto integrado por todos los individuos que tienen una cierta característica en común, tal como el lugar de nacimiento, el idioma, determinados rasgos étnicos o la pertenencia a alguna tradición cultural. La comunidad, entonces, es una mera adición que unifica la pluralidad de sus elementos, pero sin agregarles ningún rasgo nuevo que los constituya en una realidad autónoma e independiente de sus características fácticas.

Dicho de otra manera, Cohen sostiene que, sin el conocimiento previo del individuo en tanto que tal, no puede accederse nunca al concepto de la totalidad de los individuos, o sea a la idea de la comunidad. Pero el individuo sería accesible directamente por medio de la sensación. Por lo tanto, la sensación debe preceder y fundamentar al pensamiento. Esta es la raíz epistemológica del prejuicio de la particularidad, que Cohen quiere desarmar, para luego poder atacar el concepto de comunidad.

Ahora bien, para criticar el prejuicio de la particularidad, es necesario recurrir a la clasificación de los tipos de juicios de la LrE. En esos textos, Cohen intenta demostrar cuál es la relación que debe existir entre el pensamiento y la sensación, si lo que se busca es producir el conocimiento puro, o sea la ciencia matemático-natural.

En realidad, la $L r E$ defiende una relación entre el pensamiento y la sensación, que es inversa a la que se da en el prejuicio de la particularidad. Cohen identifica al conocimiento con el conocimiento puro, es decir, el conocimiento que es producido exclusivamente a partir de sí mismo, sin referencia a ninguna realidad dada de antemano. El conocimiento es pura actividad, y no puede contener en sí pasividad alguna. Pero admitir que el conocimiento comienza con la sensación significaría afirmar que depende de una serie de objetos dados previamente e independientes de él, lo que es lo mismo que decir que el conocimiento no sería puro.

Por lo tanto, el conocimiento sólo comienza con el pensamiento. El último fundamento del conocimiento científico está constituido, en realidad, por los juicios de las leyes del pensamiento (die Urteile der Denkgesetze), el primero de los cuales es el juicio del origen. ${ }^{63}$

${ }^{63} \operatorname{LrE}, 79-93,104,117,124-127,137,179$ ss., 237, 393, 455, 583 ss., 587 ss. Las vicisitudes en la interpretación de este principio pueden seguirse en Stolzenberg, (1995), y Mayerhofer, (2004). 
Los juicios de las leyes del pensamiento son juicios constitutivos del conocimiento puro, porque determinan la calidad del pensamiento científico, es decir, las reglas que debe cumplir para no dejar de ser tal. La sensación, en cambio, pertenece a la clase de los juicios de la metodología, que simplemente regulan los pasos que deben seguirse en el procedimiento de la investigación científica. ${ }^{64}$ Un momento fundamental de los juicios de la metodología es la medición de la magnitud intensiva, que es el objetivo del juicio de la realidad efectiva (Das Urteil der Wirklichkeit). ${ }^{65}$ Por lo tanto, la sensación juega un papel secundario en la teoría coheniana del pensamiento puro. Por esta razón, Cohen rechaza el prejuicio de la particularidad y con él el concepto de comunidad, porque éste se funda en aquél. Este es el motivo de la siguiente afirmación

\begin{abstract}
La auténtica desgracia de la lógica consiste en que no se quiere dejar valer a la particularidad como un problema exclusivamente metodológico. Por eso se desliza subrepticiamente la particularidad y se desplaza al pensamiento del conocimiento. Por un lado, se plantea que el pensamiento no podría producir ni indagar la particularidad; para eso sería necesaria la sensación; por otro lado, se dice que la sensación no necesitaría del pensamiento para la particularidad; para ello estaría la sensación, y ella sería suficiente. Toda oposición contra el idealismo y su suficiencia se enraíza en este error; en la caracterización lógica de la particularidad. ${ }^{66}$
\end{abstract}

Este error metodológico cobra relevancia moral si se lo aplica a la discusión del concepto de comunidad, porque pone en evidencia su imposibilidad de abarcar a todos los sujetos que quieran participar de ella. Si se aparta al pensamiento de su función fundamentadora y se coloca en su lugar a la sensación, entonces el concepto de comunidad puede sustituir al de persona jurídica, pero también desaparecerá la idea de una voluntad común a todos sus miembros, para dar lugar a una relación de mera adscripción. Es decir, los miembros de la comunidad no pertenecen a ella a causa de su cumplimiento de determinadas reglas comunes a todos, sino más bien en base a la posesión de ciertas características que pueden establecerse fácticamente.

Según Cohen, en el patriotismo es posible advertir "el déficit de universalidad" del concepto de comunidad, porque esta posición lleva a que el individuo se cierre en la particularidad de su nación, es decir, del conjunto de quienes comparten determinadas características, y excluye, como si fueran sus enemigos, a quienes no pertenecen a ella. ${ }^{67}$

${ }^{64}$ LrE, 404-616. Los juicios de la matemática y la ciencia matemático-natural contienen, en la terminología de Cohen, "naiven Kategorien", es decir, categorías que proveen de los fundamentos para la constitución del objeto de la experiencia científica ( $L r E, 431)$. Los juicios de la metodología, en cambio, permiten dar un paso más y establecer las condiciones de la tarea de la investigación científica ( $L r E, 429)$. Esto es, en los juicios de la metodología se trata del modo en que la ciencia obtiene nuevos conocimientos y amplía su horizonte.

${ }^{65}$ LrE, 454-501.

66 "es ist das eigentliche Unglück der Logik, dass man die Einzelheit nicht lediglich als ein Problem der Methodik gelten lassen will. Daher schleicht sich die Empfindung ein und verdrängt das Denken der Erkenntnis. Einesteils meint man, das Denken könne die Einzelheit nicht ergeben und nicht ermitteln; dazu bedürfe es der Empfindung; andernteils meint man es aber so, dass es für die Einzelheit des Denkens nicht bedürfe; dazu sei eben die Empfindung da; und die allein zulänglich. So wurzelt aller Gegensatz gegen den Idealismus und seine Zulänglichkeit in diesem Fehler; in der logischen Charakteristik der Einzelheit." (ErW, 234).

${ }^{67}$ Ver. la cuestión del colectivismo en: Günther, (1971), 159. 
Otro error que suelen cometer quienes defienden el concepto de comunidad frente al de persona jurídica, es afirmar que la comunidad es más apta para representar al sujeto universal de la ética, porque se funda en una relación fáctica entre los sujetos, que es armónica y los cohesiona fuertemente. ${ }^{68}$ En esta concepción juega un rol importante la analogía con la comunidad religiosa, signada por la armonía entre sus miembros, o sea la idea un regnum gratiae. ${ }^{69}$ Cohen argumenta que, por el contrario, los grupos sociales caracterizados por la idea de la comunidad -tales como la familia, el pueblo, la clase social, etc.- suelen estar signados por el antagonismo y el conflicto entre ellos mismos.

Cohen piensa en las rivalidades entre las familias, en las luchas de clase, o en los conflictos entre las mayorías y minorías raciales o religiosas, que conocía de cerca, por pertenecer a una familia judía de la Alemania decimonónica. ${ }^{70}$ La unidad de la comunidad está siempre en peligro por los enfrentamientos que su existencia misma conlleva. Cuando el individuo se integra en una comunidad no suele dejar de lado su egoísmo, para ponerse al servicio de objetivos comunes, sino que, antes bien, estos objetivos pretendidamente colectivos son un disfraz de su autointerés.

El modelo de la comunidad se ha mostrado entonces poco idóneo para dar cuenta de la autoconciencia, como una forma de subjetividad universal, que pone en relación a cada individuo con todos los demás por medio de leyes elaboradas por todos.

\section{El Estado como persona jurídica}

Una vez que Cohen ha rechazado el concepto de comunidad para fundamentar el concepto de Estado, recurre a la figura de la persona jurídica. Esta figura legal consiste en una voluntad artificial, es decir, puramente jurídica, que es análoga a una persona física, porque puede realizar muchas operaciones similares, tales como comprar, vender, endeudarse, premiar determinados comportamientos y castigar otros, etc. ${ }^{71}$ Pero lo característico, desde el punto de vista de Cohen, es la capacidad que tiene de regular sus acciones por medio de disposiciones establecidas por sus mismos integrantes. ${ }^{72}$ El modelo de persona jurídica que Cohen tiene en

${ }^{68}$ Estos argumentos de Cohen ganan mucha actualidad si se los considera en relación con el debate entre universalistas y comunitaristas morales.

${ }^{69}$ Ver. $\operatorname{ErW}, 238$.

${ }^{70} \mathrm{ErW}, 241$.

${ }^{71} \mathrm{ErW}, 229$. Ver Günther, (1971), 135. El concepto que se utilizaba para expresar esta idea en Europa antes del siglo XIX era el de persona moral (por ejemplo, en Puffendorf). Otras expresiones que se utilizaban para dar a entender la naturaleza no sensible de la persona jurídica corporativa eran: persona ficta, persona repraesentata, persona intellectualis, persona imaginaria, persona artificialis, persona mystica o persona composita (Winter, 1980, 318).

${ }^{72}$ La idea de una democracia económica ha ejercido influencia en algunos teóricos contemporáneos. Por ejemplo, Van der Linden considera pertinente la aplicación del imperativo categórico a la democratización de la toma de decisión en las empresas, en consideración de la eliminación de muchos de los males que provoca la estructura corporativa de las empresas. Por ejemplo, se solucionarían o mejorarían muchas situaciones que los obreros nunca consentirían, tales como un sistema de salud deficiente, los bajos sueldos, las políticas de privacidad invasivas, etc. Del mismo 
mente es el de la cooperativa, considerada por Gierke como perteneciente a la tradición propia del derecho alemán. ${ }^{73}$

La cooperativa consiste en la voluntad de todos los cooperantes expresa en el documento del Estatuto, que regula el modo en que se votará en el futuro, es decir el modo en que esta voluntad común misma se expresará de allí en adelante. Esta voluntad es universal porque se aplicará a todos aquellos miembros que la han producido. Pero también es necesaria, porque no puede ser modificada caprichosamente a causa de algún cambio azaroso en las voluntades empíricas de los cooperantes, sino que más bien debe seguir una serie de procedimientos, que están expresados en el Estatuto mismo.

Es decir que la persona jurídica cumple con los requisitos de universalidad y necesidad que Cohen reclama para la ética como disciplina filosófica, y por lo tanto para su concepto del Estado. De este modo, la persona jurídica juega un rol modélico en la teoría del Estado de Cohen, y le permite darle un carácter idealista y kantiano, es decir germano y judío a la vez, según vimos al comienzo de este trabajo. Utilizando esta figura legal, Cohen defiende la idea de un Estado en el cual cada ciudadano participe en la legislación por medio de la actividad de sus representantes en el Parlamento. Se trata de la idea de un socialismo kantiano o ético, en la línea del ala revisionista de la socialdemocracia alemana liderada por Eduard Bernstein. ${ }^{74}$

La ciencia del derecho entonces es aquel ámbito de la cultura en el que se manifiesta la autoconciencia, es decir aquella subjetividad en la que están inextricablemente unidos cada individuo con la universalidad de la ley moral, que básicamente consiste en respetar el derecho de los demás a autolegislarse y participar en la persona jurídica del Estado. ${ }^{75}$ La categoría de la autoconciencia exige necesariamente la inclusión de

modo, las empresas deberían incorporar en su toma de decisiones a los miembros de la comunidad, en caso de que éstas afecten su vida, provocando contaminación ambiental, destruyendo el patrimonio histórico de la comunidad, así como otra clase de prejuicios (Van der Linden, 1998, 220-221). Otros beneficios que Van der Linden considera que se desprenderían de una democracia empresarial serían el bajo incentivo que tendrían las empresas para expandirse continuamente, porque sus ganancias no aumentarían necesariamente, dado que la expansión implicaría la contratación de más empleados y la necesidad de repartir las ganancias con ellos. Esto implicaría que las empresas tendrían en realidad poco tamaño, lo cual es indispensable para la puesta en práctica de una democracia deliberativa en la institución que sea. La división de las ganancias entre los empleados disminuiría el afán de lucro de las empresas en general, y podría traer como consecuencia que los empleados se tomaran más tiempo libre y trabajaran un poco menos, lo que jamás se podría esperar en una corporación dirigida por algunos ejecutivos. Asimismo, las decisiones de la empresa tomarían en cuenta cada vez más las necesidades del ciudadano promedio, dado que serían hombres de esta clase social quienes tomarían las decisiones fundamentales. Es decir, si se contamina el medio ambiente las consecuencias no las sufrirá seguramente el ejecutivo que puede comprarse una casa en una zona de buena calidad de vida, sino más bien el empleado que no tiene mucho poder de decisión a la hora de elegir una vivienda (Van der Linden, 1998, 229-230).

${ }^{73}$ Remito al análisis de Winter, (1980), 299-310, que aún no ha sido superado hasta el presente.

${ }^{74}$ Cohen consideró ya desde sus primeros escritos a Kant como el verdadero fundador del socialismo alemán (EmkN, 112). Ver. el comentario de Meyer en (1993), 261.

${ }^{75}$ Acerca de la recepción en la $E r W$ de la idea kantiana de la humanidad como fin en sí mismo, Ver Van der Linden, (1994), 149-155. 
todas las minorías en la toma de decisiones propia de la vida estatal. De este modo, también la minoría judía queda resguardaba de toda forma de maltrato y coacción arbitraria en el ideal de Estado que Cohen propone, considerado como la culminación de la integración entre la germanidad y el judaísmo.

\section{Bibliografía}

\section{Abreviaturas utilizadas}

DuJ: Cohen, H., (1915), “Deutschtum und Judentum”, en: (1924), Hermann Cohens Jüdische Schriften, Berlin, 2. Band, Schwetschke \& Sohn, pp. 237-301.

EmkN: Cohen, H., (1914), Einleitung mit kritischem Nachtrag, Leipzig, Verlag von Friedrich Brandstetter.

ErW: Cohen, H., (1907), Ethik des reinen Willens, en: (2002), Herman Cohen. Werke, herausgegeben vom Hermann-Cohen-Archiv am Philosophischen Seminar der Universität Zürich unter der Leitung von Helmut Holzhey, Band 7, System der Philosophie. 1. Teil. Ethik des reinen Willens, Georg Olms Verlag, Hildesheim-ZürichNew York. Einleitung von Peter A. Schmid. Nachdruck der 2. revidierten Auflage, (Berlin, Bruno Cassirer, 1907).

KTE: Cohen, H., (1918), Kants Theorie der Erfahrung, en: (1987), Herman Cohen. Werke, herausgegeben vom Hermann-Cohen-Archiv am Philosophischen Seminar der Universität Zürich unter der Leitung von Helmut Holzhey, Band 1.1, Text der dritten Auflage 1918 und Einleitung von Geert Edel; Georg Olms Verlag, Hildesheim-ZürichNew York. Einleitung von Helmut Holzhey. Nachdruck der 3. Auflage (Berlin, Bruno Cassirer, 1919).

LrE: (1914), Logik der reinen Erkenntnis, en: (2005), Herman Cohen. Werke, herausgegeben vom Hermann-Cohen-Archiv am Philosophischen Seminar der Universität Zürich unter der Leitung von Helmut Holzhey, Band 6, System der Philosophie. 1. Teil. Logik

der reinen Erkenntnis, Georg Olms Verlag, Hildesheim-Zürich-New York. Einleitung von Helmut Holzhey. Nachdruck der 2. verbesserten Auflage (Berlin, Bruno Cassirer, 1914).

\section{Bibliografía Secundaria}

Geismann, G., (1993), “Der Berliner Antisemitimusstreit und die Abdankung der rechtlich-praktischen Vernunft", en: Kant-Studien, 83. Jahrg., pp. 369-380.

Günther, H., (1971), System und Fortschritt im Denken Hermann Cohens, Köln, Dissertation.

Holzhey, H., (1993), “Hermann Cohen: der Philosoph in Auseinandersetzung mit dem politischen und gesellschaftlichen Probleme seiner Zeit", in: Brandt, R.., 
Orlik, F., (Hrsg.), (1993), Philosophisches Denken - Politisches Wirken. Hermann Cohens Kolloquium Marburg 1992, Hildesheim-Zürich-New York, Georg Olms Verlag, pp.15-36.

Lembeck, K.-H., (1994), Platon in Marburg. Platonrezeption und Philosophiegeschichtsphilosophie bei Cohen und Natorp, Würzburg, Königshausen \& Neumann.

Lübbe, H., (1958), "Die politische Theorie des Neukantianismus und des Marxismus", in: Holzhey, H., (Hrsg.), (1994), Auslegungen. Band 4. Hermann Cohen, Frankfurt am Main-Berlin-Bern-New York-Paris-Wien, Peter Lang, pp.229-246.

Lübbe, H., (1963), "Neukantianische Sozialismus", in: Politische Philosophie in Deustchland. Studien zu ihrer Geschichte, Basel, Schwabe Verlag, SS.85-125. In: Ollig, H.L., (Hrsg.), (1987), Materialen zur Neukantianismus Diskussion, Darmstadt, Wissenschaftliche Buchgesellschaft, pp.219-263.

Mayerhofer, H., (2004), Der philosophische Begriff der Bewegung in Hermann Cohens „Logik der reinen Erkenntnis", Wien, WUV, Universitätsverlag.

Meyer, T., (1993), “Eine unzeitgemäße Intervention, die an der Zeit war. Hermann Cohens neukantianischer Sozialismus und die sozialdemokratische Ideologie im Kaiserreich", in: Brandt, R.., Orlik, F., (Hrsg.), (1993), Philosophisches Denken Politisches Wirken. Hermann Cohens Kolloquium Marburg 1992, Hildesheim-ZürichNew York, Georg Olms Verlag, pp.257-269.

Ollig, H. L., (1979), Religion und Freiheitsglaube, Forum Academicum in der Verlagsgruppe Athenäum, Hain, Scriptor, Hanstein.

Palmer, G., (2006), "Judaism as a ,method' with Hermann Cohen and Franz Rosenzweig“, en: Gibbs, R., (eds.), (2006), Hermann Cohen's Ethics, Leiden-Boston, Brill, pp. 37-63.

Schwarzschild, S., (1956), "The Democratic Socialism of Hermann Cohen", in: Holzhey, H., (Hrsg.), (1994), Auslegungen. Band 4. Hermann Cohen, Frankfurt am Main-BerlinBern-New York-Paris-Wien, Peter Lang, pp.205-227.

Sieg, U., (1994), Aufstieg und Niedergang des Marburger Neukantianismus. Die Geschichte einer philosophischen Schulgemeinschaft, Würzburg, Könighausen \& Neumann.

Stolzenberg, J., (1995), Ursprung und System. Probleme der Begründung systematischer Philosophie im Werk Hermann Cohens, Paul Natorps und beim frühen Martin Heidegger, Göttingen, Vandenhoeck \& Ruprecht.

Van der Linden, H., (1994), "Cohens sozialistische Rekonstruktion der Ethik Kants", en: Holzhey, H., (comp.), Ethischer Sozialismus, Frankfurt, Suhrkamp, pp.146-165. 
Van der Linden, H., (1998), „,A Kantian Defense of Enterprise Democracy“, en: Kneller, J., Axinn, S., (eds.), (1998), Autonomy and Community. Readings in Contemporary Kantian Social Philosophy, New York, State University of New York Press, pp. 213-237.

Wiedebach, H., (1997), Die Bedeutung der Nationalität für Hermann Cohen, HildesheimZürich-New York, Georg Olms Verlag.

Winter, E., (1980), Ethik und Rechtswissenschaft. Eine historisch-systematische Untersuchung zur Ethik-Konzeption des Marburger Neukantismus im Werke Hermann Cohens, Berlin, Duncker \& Humblot.

Zank, M., (2006), “The ethics in Hermann Cohen's philosophical system”, en: Gibbs, R., (eds.), (2006), Hermann Cohen's Ethics, Leiden-Boston, Brill, pp. 1-15.

Recibido: Mayo 24 de 2009 - Aprobado: Agosto 15 de 2009 